\title{
The role of interactions of nucleic acids with lipids in nuclear pore assembly, genome expression and carcinogenesis
}

\begin{abstract}
DNA-lipid (membrane) interactions, which had a peak in popularity in 1970-1985, were subsequently replaced by the nuclear matrix and interactions of DNA-cationic amphiphiles. The nuclear matrix is considered now an artifact structure, and DNA complexes with cationic lipids (lipoplexes) from the hope of gene therapy have become a complete disappointment. Therefore, it's time to look at DNA-lipid interactions in the light of modern data and methods and try to understand their role in cellular structures and functions. The author suggests a new model of nuclear pore based on DNA/RNA-lipid interactions, the disclosure of R-loop (DNA/RNA hybrid) in the lipid environment and the formation of an opening between two lipid bilayers without the participation of proteins. The emergence of ssDNA after the disclosure of the triple helix R-loop suggests initiation of transcription to ssDNA, which explains the increased transcription in the nuclear pore region. The author analyzes all components of nuclear pores capable of influencing transcription, and consequently, cancerous degeneration of cells. These are nucleoporins, as well as low-molecular RNA (snRNA, lncRNA), which form R-loop with chromatin DNA.
\end{abstract}

Keywords: DNA-cationic amphiphiles, molecular biology, nuclear matrix, nuclear pores, heterochromatin
Volume 7 Issue 4 - 2018

\author{
Vasily Kuvichkin \\ Institute of Cell Biophysics, Russian Academy of Sciences, Russia
}

Correspondence: Kuvichkin Vasily, Institute of Cell Biophysics, Russian Academy of Sciences, Russia,

Email vvkuvichkin@gmail.com

Received: July 30, 2018 | Published: August 06, 2018

\section{Introduction}

In our days when the methods of molecular genetics are dominant in biology, traditional biochemistry and biophysics rank low and serve as Cinderella in molecular biology. Unfortunately, biochemists and biophysicist do not have enough time to estimate new methods (protocols) that emerged in molecular biology and genetics. The demerits of the methods are multiplied giving birth to other errors. The example of it is an approximately thirty-year investigation of nuclear matrix, which according to recent data does not exist in the structure of nucleus! Furthermore, a lot of valuable ideas which were rejected because of the absence of appropriate biochemical methods in the past have not been recovered and don't got a development after the appearance of the techniques which could be used to explain their correctness. One of the undeservingly forgotten ideas, which was exploited during investigation within $70 \mathrm{~s}-80 \mathrm{~s}$ in the past century, was the conception of DNA-membrane complexes, ${ }^{1}$ and as a particular case, DNA-lipid interactions.

\section{DNA-lipids interactions: fifty years ago}

Our study on DNA-lipid interactions in vitro, ${ }^{2}$ has been undertaken in $1976,{ }^{3}$ and continues until present in collaboration with many laboratories abroad. ${ }^{4}$ Ternary complexes (TC): DNA-zwitterionic lipids in the presence of divalent metal cations were explored almost at the same time at our laboratory and by Budker VG. ${ }^{5}$ The interest in investigation of TC was promoted by offering our first model of $\mathrm{TC}$ formation and the probable involvement of TC in the formation of nuclear pores. ${ }^{6}$ According to the current nuclear pore models, lipids and DNA do not participate in them formation. ${ }^{7}$ In the author's opinion, this major disadvantage does not allow an understanding of how nuclear pores can built. It was recently discovered that chromatin areas near nuclear pores have increased transcriptional activity. ${ }^{8,9}$

\section{$\mathbf{R}$-loop in vitro and in vivo}

The most logical explanation for these data suggested in our nuclear pore model assumes three-stranded DNA $\backslash$ RNA hybrids (R-loops) participation Figure 1. ${ }^{10,11}$ In the nucleus, DNA unwinds in specific regions of heterochromatin (three-stranded DNA/RNA hybrids), which determines the specificity of open regions in DNA (formation of so-called R-loops). Furthermore, R-loop opening is observed at temperatures $20-30^{\circ} \mathrm{C}$ degrees lower than that at which opening of the DNA helix with the same nucleotide sequence takes place. ${ }^{11}$ We supposed that low molecular weight nuclear RNA lmwRNA, such as C (U1) and D (U2), with lengths of 186nt and $167 \mathrm{nt}$ in length, respectively, are candidates for R-loop formation. ${ }^{12}$ With the discovery of a large group of long non-coding RNAs, ${ }^{13}$ a portion of these RNAs around of 200nt in size can be referred to as C-and D-type lncRNA by virtue of their ability to form R-loops.

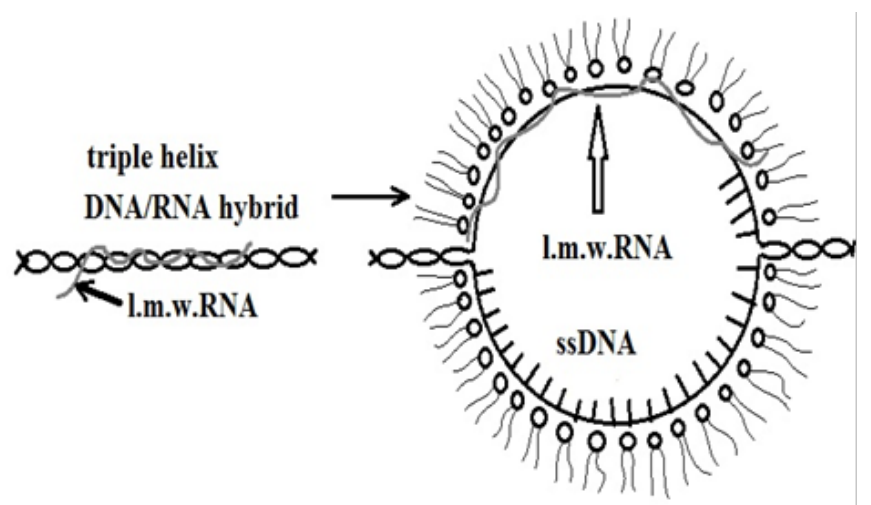

Figure I Opening of DNA/RNA triplex during nuclear pore formation. 


\section{Two type vesicles and $\mathbf{R}-$ loop need for nuclear pore assembly}

We have suggested that R-loops form during TC formation in the nucleus from chromatin and two Small Unilamellar Vesicles (SUVs) of $70 \mathrm{~nm}$ in diameter, from which nuclear pores are formed. ${ }^{14}$ SUVs do not fuse with each other; however, when interacting with DNA in the presence of $\mathrm{Mg}^{2+}$ cations, meaning that they are able to form TCs vesicles belted by ssDNA Figure $2(\mathrm{~A}-\mathrm{C})$. So, we have rather simple for biophysics but complex for biologists model based on the channel formation between two lipid bilayers. This model has been developed taking into account DNA-induced fusion of SUV (TC formation) and $\mathrm{Mg}^{2+}$ induced fusion of TC bilayer with two lipid bilayers of liposomes (in vitro) / vesicles (in vivo) with pore formation between these bilayers Figure $2(\mathrm{D}-\mathrm{G}) .{ }^{16}$ This model reflects a scheme of TC formation with chromatin through DNA-induced fusion of small membrane vesicles and $\mathrm{Mg}^{2++}$ induced fusion of the formed TC with double lipid bilayer which is formed out of large $((>400 \mathrm{~nm})$ liposomes or by another version out of the endoplasmic reticulum . The results reported in, ${ }^{17}$ show that nuclear pores have not been formed until the nuclear envelope is completely closed. This condition can significantly affect the energy of surface tension of nuclear membrane and is crucial for our model of the formation of nuclear pores. The involvement of nuclear proteins-fusogenes is admissible but not obligatory at this stage of the formation of the nuclear envelope as was shown in our previous study. ${ }^{16}$ Substances, that have an effect on the energy of the surface tension, should also affect the structure and the amount of the pores that are formed. We assumed that proteinsnucleoporins participate in the formation of the native structure of the pore after channel formation between nuclear membranes, but, most probably, nucleoporins are bound to the elements of R-loop just during the formation of TC (pre-pores). Then, these proteins are part of transcriptional factories or nuclear pores, providing a basis for attachment of other nucleoporins.

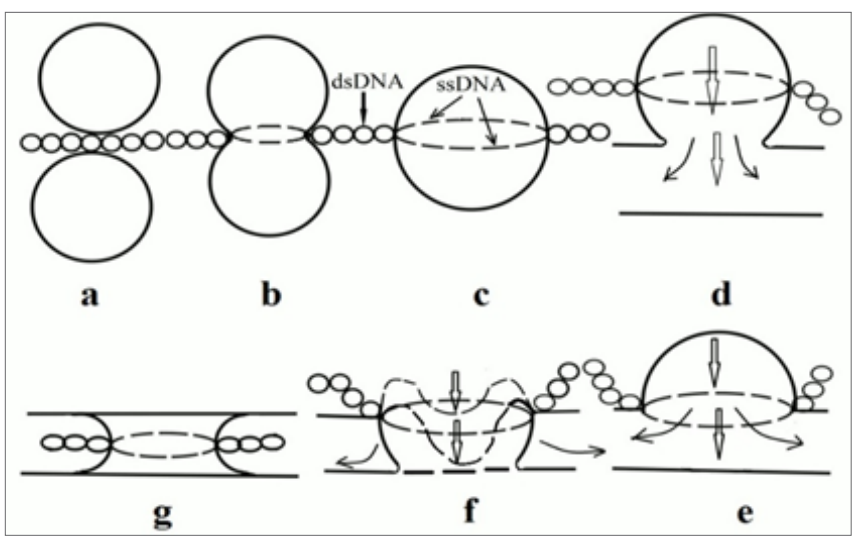

Figure 2 The nuclear pore assembly model (I5) based on the TCs (C) formation $(A-C)$ and subsequent their fusion with double bilayer $(D-F)$ and pore formation $(G)$.

\section{Carcinogenesis seems easy for elucidation from point of view our nuclear pore model}

A link between some nucleoporins and cancer is known Figure 3. ${ }^{(17,18)}$ In order to explain this connection it is possible to accept the point of view of the said authors or to consider the above stated interaction between nucleoporins and the elements of open R-loop
(ssDNA and DNA/RNA hybrid). Any changes to interaction between nucleoporins and these elements will lead to alteration in the level of transcription of neighboring genes that may be a cause of cancer. Currently, there are plenty of studies reporting about a direct link of lncRNA, snRNA to cancer occurrence or about inhibition of tumor formations by the fragments or derivatives of these RNA. ${ }^{19,20}$ This has been already discussed in our works 15 years ago. Exchange of low molecular RNA between normal and cancer cells resulting new nuclear pore assembly may result in transformation normal cell to cancerous. ${ }^{21}$ The presence of ssDNA in the nuclear pore where transcription in the nucleus is initiated suggests that enzyme S1 nuclease can be used in cancer therapy.

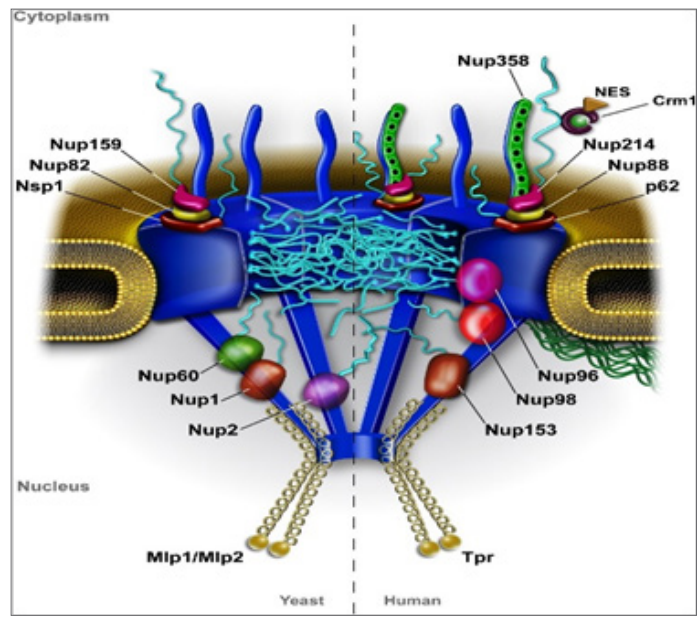

Figure 3 The nucleoporins wish have relation to cancer (I8).

\section{Acknowledgements}

None.

\section{Conflict of interest}

The author declares no conflict of interest.

\section{References}

1. Moyer MP. The association of DNA and RNA with membranes. Int Rev Cytol. 1979;61:1-61.

2. Shabarchina LI, Sukhorukov BI, Kuvichkin VV, et al. Infrared spectroscopic study of DNA-lipid interactions. DNA compacting on disperse particles. Biofizika. 1979;24(6):990-998.

3. Kuvichhkin V.V. Lipid-nucleic acids interactions in vitro and in vivo. Bioelectrochemistry. 2002;58(1):3-12.

4. Süleymanoğlu E. $\mathrm{Mg}^{2+}$ induced DNA compaction, condensation, and phase separation in gene delivery vehicles based on zwitterionic phospholipids: a dynamic light scattering and surface-enhanced Raman spectroscopic study. J Biol Inorg Chem. 2017;22(8):1165-1177.

5. Budker VG, Godovikov AA, Naumova LP, et al. Interaction of polynucleotides with natural and model membranes. Nucleic Acids Res. 1980;8(11):2499-2515.

6. Kuvichkin VV. Theoretical model of DNA-membrane contacts. Biofizika. 1983;28(5):771-775.

7. Hoelz A, Glavy JS, Beck M. Toward the atomic structure of the nuclear pore complex: when top down meets bottom up. Nat Struct Mol Biol. 2016;23:624-630. 
8. Taddei A. Active genes at the nuclear pore complex. Curr Opin Cell Biol. 2007;19(3):305-310.

9. Arib G, Akhtar A. Multiple facets of nuclear periphery in gene expression control. Curr Opin Cell Biol. 2011;23(3):346-353.

10. $\mathrm{Xu} \mathrm{W}, \mathrm{Xu} \mathrm{H}, \mathrm{Li} \mathrm{K}$, et al. The R-loop is a common chromatin feature of the Arabidopsis genome. Nat Plants. 2017;3(9):704-714.

11. Darby RA, Sollogoub J, Keen C, et al. High throughput measurement of duplex, triplex and quadruplex melting curves using molecular beacons and a Light Cycler. Nucleic Acids Res. 2002;30(9):1-39.

12. Pederson T, Bhorjee JS. Evidence for a role of RNA in eukaryotic chromosome structure. Metabolically stable, small nuclear RNA species are covalently linked to chromosomal DNA in He La cells. $J$ Mol Biol. 1979;128(4):451-480.

13. Taylor DH, Chu ET, Spektor R, et al. Long non-coding RNA: regulation of reproduction and development. Mol Reprod Dev. 2015;82(12):932-956.

14. Vigers GPA, Lohka MJA. Distinct vesicle population targets membranes and pore complexes to the nuclear envelope in Xenopus eggs. J Cell Biol. 1991;112(4):545-556.
15. Kuvichkin VV. The mechanism of a nuclear pore assembly: a molecular biophysics view. J Membr Biol. 2011;241(3):109-116.

16. Taylor DH, Chu ET, Spektor R, et al. Long non-coding RNA: regulation of reproduction and development. Mol Reprod Dev. 2015;82(12):932-956.

17. Xu S1, Powers MA. Nuclear pore proteins and cancer, Semin. Cell Dev Biol. 2009;20(5):620-630.

18. Alwin Kohler, Hurt ED. Gene Regulation by Nucleoporins and Links to Cancer. Molecular Cell. 2010;38(1):6-15.

19. Jens Köhler. Circulating U2 small nuclear RNA fragments as a diagnostic and prognostic biomarker in lung cancer patients. J Cancer Res Clin Oncol. 2016;142(4):795-805.

20. Heeyoun Bunch. Gene regulation of mammalian long non-coding RNA. Mol Genet Genomics. 2018;293(1):1-15.

21. Cancer Biology and the Nuclear Envelope. Recent Advances May Elucidate Past Paradoxes. In: Eric C, Schirmer Jose I, de las Heras, editors. Advances in Experimental Medicine and Biology. 2014. 